\title{
Controlling the Chaos of Logistic Map using Switching Strategy
}

\author{
Sudesh Kumari, Renu Chugh, Ashish Nandal
}

\begin{abstract}
In our very recent work (2019), we extended the stability performance of logistic map up to a higher value of $r$ using SP orbit. In this article, we further extend this range of stability by adopting switching strategy (Parrondo's Paradox) of controlling the chaos of dynamical systems. We observe that even the earlier chaotic orbits of four step feedback procedure can be converted into periodic orbits. Our approach can be used to solve a wider circle of engineering problems.
\end{abstract}

Keywords: SP orbit, switching strategy, logistic map, bifurcation plot.

\section{INTRODUCTION}

The dynamical systems are very sensitive on the initial conditions, i.e, it is impossible to forecast the long term behavior of the dynamical systems. The logistic map occupied a renowned position in understanding the complex dynamical systems due to its simplest form. It was originally introduced by P.F. Verhulst [1] to study the population of non- overlapping generation. The relation between the two generations, i.e, new $\left(x_{n+1}\right)$ and old $\left(x_{n}\right)$ is represented by the logistic map as

$$
x_{n+1}=r x_{n}\left(1-x_{n}\right), n=0,1,2, \ldots,
$$

where $r>0$ and $x_{n} \in[0,1]$. One may refer the work in [2], [3] to know more about the scientific applications of logistic map.

In 1996, Spanish physicist Juan Parrondo [4] investigated Parrondo's paradox (also known as switching strategy), which is a famous paradox in game theory. Parrondo's paradox implies that two losing games can produce a winning game when played alternatively [5]. Thereafter, a sequence of papers on Parrondo's paradox and its applications has been published (see, [6] and references therein). The one decade work of Parrondo's paradox had been summarized by Abbott [7]. Moreover, authors [8], [9] extended the concept of "lose $1 "+$ "lose $2 "=$ "win" to "chaos1"+ "chaos2"=

Revised Manuscript Received on December 30, 2019.

* Correspondence Author

Sudesh Kumari, Assistant Professor, Department of Mathematics, Government College for Girls Sector 14, Gurugram-122001, India.

E-mail: tanwarsudesh10@gmail.com

Renu Chugh, Professor, Department of Mathematics, Maharshi Dayanand University, Rohtak-124001, India. E-mail: chugh.r1@gmail.com Ashish Nandal, Assistant Professor, Department of Mathematics, Pt. NRS Government College, Rohtak-124001, India. E-mail: ashishnandalmdu@gmail.com

(C) The Authors. Published by Blue Eyes Intelligence Engineering and Sciences Publication (BEIESP). This is an open access article under the CC BY-NC-ND license (http://creativecommons.org/licenses/by-nc-nd/4.0/) "periodic". In 2019, the performance of logistic map was further extended by Kumari et al. [10], [11] using SP orbit.

Almedia et al. [8] combined the two nonlinear quadratic maps and then used Parrondo's paradox. Peacock et al. [12], [13] considered the case where "undesirable1"+ "undesirable2"= "desirable" dynamical behaviors. Further, Yadav et al. [14], [15], controlled the chaotic behavior of superior logistic map with the help of Parrondo's paradox. In this article, we shall apply the Parrondo's paradox to control the chaotic behavior of logistic map considered in SP orbit [16]. Further, we show that the combination of two chaotic orbits can be converted into a periodic orbit by iterating alternatively. Bifurcation and time series analysis have been adopting to analyze the switching strategy applied on logistic map.

\section{PRELIMINARIES}

Definition 2.1 [2] A fixed point $z_{0} \in Z$ of a self map $F: Z \rightarrow Z$ is the point for which $F\left(z_{0}\right)=z_{0}$.

Definition 2.2 [2] A point $z_{0} \in Z$ is said to be periodic point of a self map $F: Z \rightarrow Z$ with period $q$ if $F^{q}\left(z_{0}\right)=z_{0}$ where $q \in \mathbb{N}$.

Definition 2.3 [16] Let $Z \subseteq \mathbb{R}$ be a nonempty and $F: Z \rightarrow Z$. Then construct the sequence $\left\{x_{n}\right\}$ of iterates for initiator $x_{0} \in Z$ in such a way that

$$
\begin{aligned}
x_{n+1} & =\left(1-\lambda_{n}\right) y_{n}+\lambda_{n} F y_{n}, \\
y_{n} & =\left(1-\mu_{n}\right) z_{n}+\mu_{n} F z_{n}, \\
z_{n} & =\left(1-v_{n}\right) x_{n}+v_{n} F x_{n}, n=0,1,2, \ldots,
\end{aligned}
$$

where $\left\{\lambda_{n}\right\},\left\{\mu_{n}\right\}$ and $\left\{v_{n}\right\}$ are sequences in $[0,1]$. This sequence $\left\{x_{n}\right\}$ is known as SP iterates (orbit). We consider $\lambda_{n}=\lambda, \quad \mu_{n}=\mu$ and $v_{n}=v$ throughout the article.

\section{FORMATION OF ALTERNATED LOGISTIC MAP IN SP ORBIT}

Let us consider two different logistic maps $g_{1}=r_{1} x(1-x)$ and $g_{2}=r_{2} x(1-x)$ where $r_{1}, r_{2}>0$ and $x \in[0,1]$. Using Definition 2.3, the alternation of the combination of the

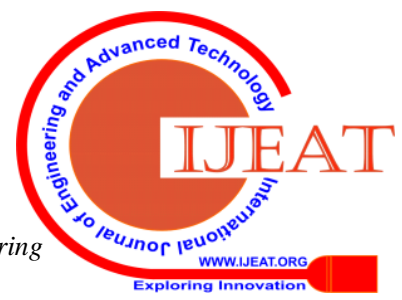


dynamics $g_{1}$ and $g_{2}$ in SP orbit can be defined as $\left(g_{1} g_{2}\right)$ :

$$
\begin{aligned}
x_{n+1} & =(1-\lambda) y_{n}+\lambda g_{1}\left(y_{n}\right), \\
y_{n} & =(1-\mu) z_{n}+\mu g_{1}\left(z_{n}\right), \\
z_{n} & =(1-v) x_{n}+v g_{1}\left(x_{n}\right), n \text { is odd; } \\
x_{n+1} & =(1-\lambda) y_{n}+\lambda g_{2}\left(y_{n}\right), \\
y_{n} & =(1-\mu) z_{n}+\mu g_{2}\left(z_{n}\right), \\
z_{n} & =(1-v) x_{n}+v g_{2}\left(x_{n}\right), n \text { is even. }
\end{aligned}
$$

Using the values of $g_{1}$ and $g_{2}$, we define the alternated logistic map in the following manner

$$
x_{n+1}=\left\{\begin{array}{l}
(1-\lambda)(1-\mu)(1-v) x_{n}+(1-\lambda)(1-\mu) v r_{1} x_{n}\left(1-x_{n}\right) \\
+(1-\lambda) \mu(1-v) r_{1} x_{n}+(1-\lambda) \mu v r_{1}^{2} x_{n}\left(1-x_{n}\right) \\
-(1-\lambda) \mu r_{1}\left\{(1-v) r_{1} x_{n}+v r_{1} x_{n}\left(1-x_{n}\right)\right\}^{2}+\lambda(1-\mu)(1-v) r_{1} x_{n} \\
+\lambda(1-\mu) v r_{1}^{2} x_{n}\left(1-x_{n}\right)+\lambda \mu(1-v) r_{1}^{2} x_{n}+\lambda \mu v r_{1}^{3} x_{n}\left(1-x_{n}\right) \\
-\lambda \mu r_{1}^{2}\left\{(1-v) x_{n}+v r_{1} x_{n}\left(1-x_{n}\right)\right\}^{2}-\lambda r_{1}\left[(1-\mu)(1-v) x_{n}\right. \\
+(1-\mu) v r_{1} x_{n}\left(1-x_{n}\right)+\mu(1-v) r_{1} x_{n}+\mu v r_{1}^{2} x_{n}\left(1-x_{n}\right) \\
\left.-\mu r_{1}\left\{(1-v) x_{n}+v r_{1} x_{n}\left(1-x_{n}\right)\right\}^{2}\right]^{2}, n \text { is odd; } \\
(1-\lambda)(1-\mu)(1-v) x_{n}+(1-\lambda)(1-\mu) v r_{2} x_{n}\left(1-x_{n}\right) \\
+(1-\lambda) \mu(1-v) r_{2} x_{n}+(1-\lambda) \mu v r_{2}^{2} x_{n}\left(1-x_{n}\right) \\
-(1-\alpha) \beta r_{2}\left\{(1-\gamma) r_{2} x_{n}+\gamma r_{2} x_{n}\left(1-x_{n}\right)\right\}^{2}+\lambda(1-\mu)(1-v) r_{2} x_{n} \\
+\lambda(1-\mu) v r_{2}^{2} x_{n}\left(1-x_{n}\right)+\lambda \mu(1-v) r_{2}^{2} x_{n}+\lambda \mu v r_{2}^{3} x_{n}\left(1-x_{n}\right) \\
-\lambda \mu r_{2}^{2}\left\{(1-v) x_{n}+v r_{2} x_{n}\left(1-x_{n}\right)\right\}^{2}-\lambda r_{2}\left[(1-\mu)(1-v) x_{n}\right. \\
+(1-\mu) v r_{2} x_{n}\left(1-x_{n}\right)+\mu(1-v) r_{2} x_{n}+\mu v r_{2}^{2} x_{n}\left(1-x_{n}\right) \\
\left.-\mu r_{2}\left\{(1-v) x_{n}+v r_{2} x_{n}\left(1-x_{n}\right)\right\}^{2}\right]^{2}, n \text { is even. }
\end{array}\right.
$$

\section{ANALYSIS OF SWITCHING STRATEGY}

Now, we apply the switching strategy or Parrondo's paradox to control the chaotic situation of logistic map in SP orbit. In our previous work [10], [11], we have shown that logistic map remains stable for a higher value of parameter $r$. To know about the chaotic range of parameter $r$ in SP orbit, we have plotted bifurcation diagram for $\lambda=\mu=v=0.9$ in Fig.

1. We observe that logistic map exhibits chaotic behavior for $r>3.85$.

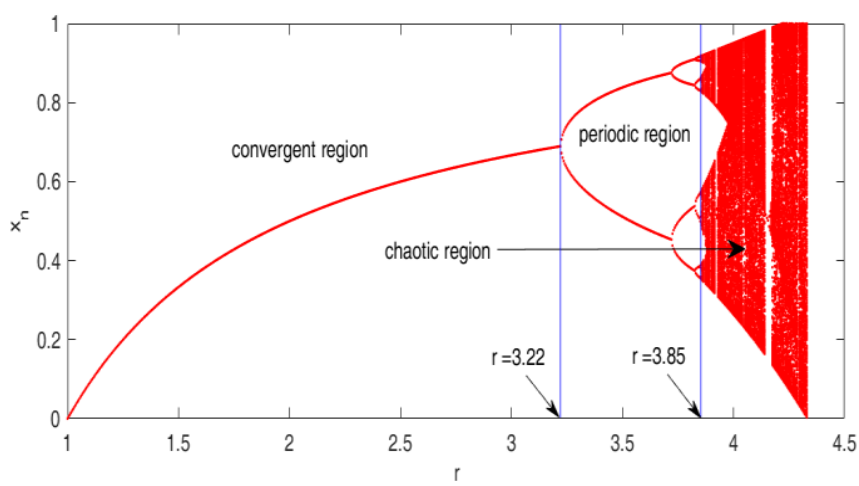

Fig. 1. Bifurcation diagram of map for $\lambda=\mu=v=0.9$
Now, to analyze the switching strategy, we depict the bifurcation plot of the system defined in (1) by fixing the value of $r_{1}$ as a chaotic value of logistic map, i.e., $r_{1}=3.80$ (see Fig. 1) for odd number of iterations and vary the value of $r_{2}$ for even number of iterations at $\lambda=\mu=v=0.9$ in Fig. 2. In Figs. 3 and 4, the bifurcation plots for chaotic values of $r_{1}=3.87$ and $r_{1}=3.89$ have been drawn. From Figs. 1, 2, 3 and 4, one can easily observe the difference between the bifurcation plots of logistic map and alternate logistic map defined in SP orbit by (1). Also, it is noticed that as we increase the chaotic value of $r_{1}$ the dynamical behavior of logistic map becomes clearer.

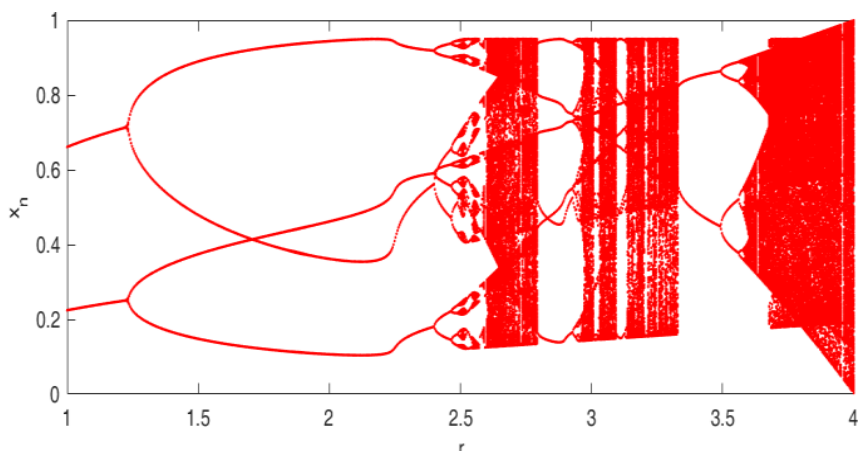

Fig. 2. Bifurcation plot for switching strategy for $\lambda=\mu=v=0.9, r_{1}=3.80$

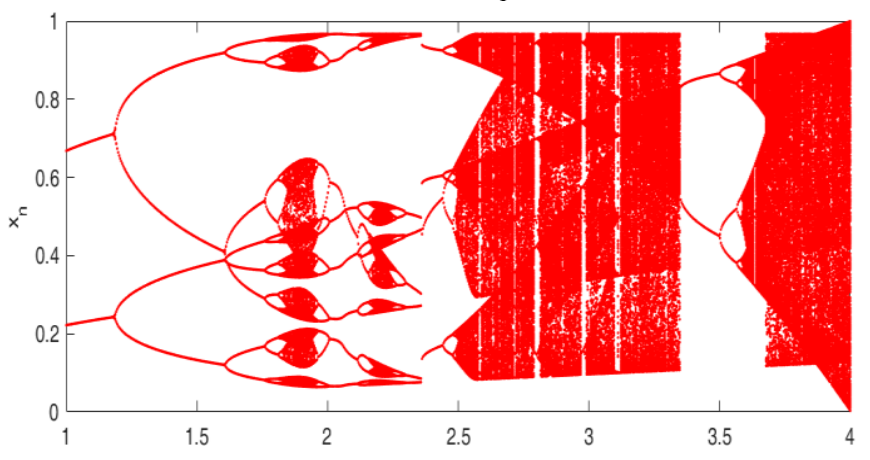

Fig. 3. Switching strategy for $\lambda=\mu=v=0.9, r_{1}=3.87$

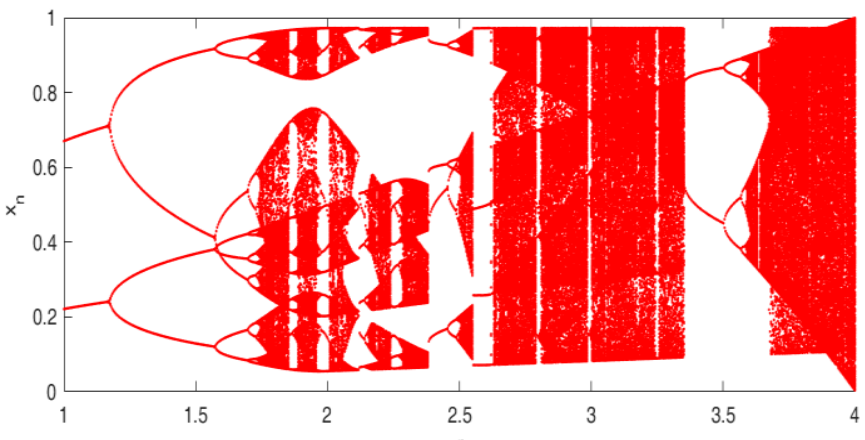

Fig. 4. Switching strategy for $\lambda=\mu=v=0.9, r_{1}=3.89$ 
Now, we apply time series analysis to show that two chaotic orbits of logistic map can be converted into a periodic orbit, i.e., the chaotic behavior can be controlled by adopting switching strategy.

We shall present several examples to demonstrate the fact that "chaotic1+chaotic 2 = periodic".

Let us take chaotic values of $r_{1}$ and $r_{2}$ from Fig. 1 to validate the fact "chaos $1+$ chaos $2=$ order" for different values of $\lambda=\mu=v$. The chaotic orbit of logistic map can be made periodic orbit of period 2, we have represented this fact by Fig. 5 for $r_{1}=3.92, r_{2}=3.94$ and $\lambda=\mu=v=0.9$. In Fig. 6 , we have shown that at two chaotic values $r_{1}=3.94$, $r_{2}=3.84$ and $\lambda=\mu=v=0.9$, the logistic map exhibits periodic behavior having period 4. Further, for $r_{1}=3.823$, $r_{2}=3.89$ and $\lambda=\mu=v=0.9$, the logistic map attains periodic orbit of period 6 as shown in Fig. 7. Similarly, at $r_{1}=4.57, r_{2}=4.02$ for $\lambda=\mu=v=0.8$, the logistic map has orbit of order 4 (see Fig. 8).

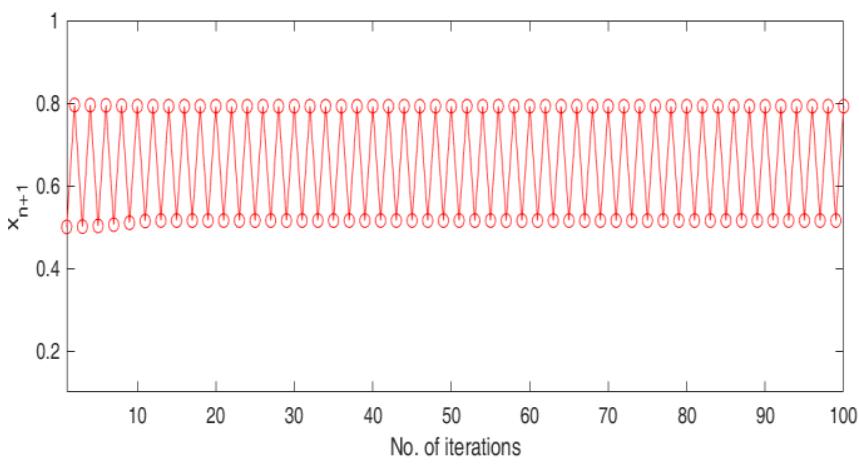

Fig. 5. Orbit of order-2 at $\lambda=\mu=v=0.9, r_{1}=3.92$ and $r_{2}=3.94$

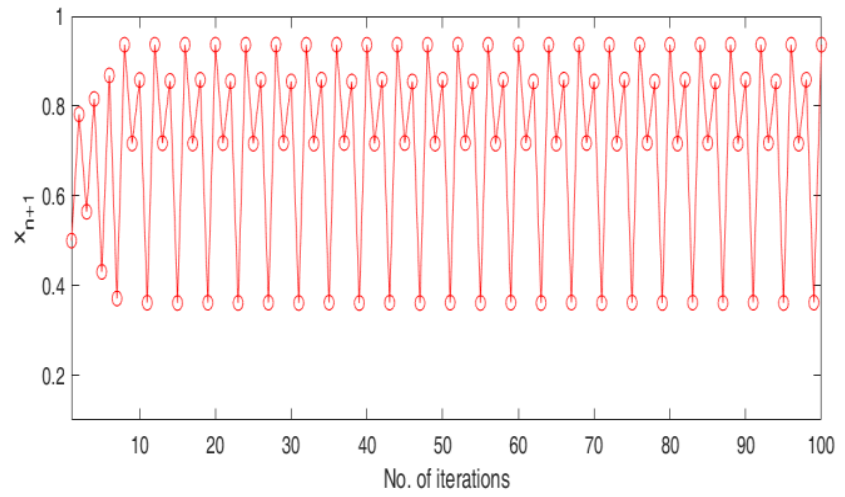

Fig. 6. Orbit of order-4 at $\lambda=\mu=v=0.9, r_{1}=3.94$ and $r_{2}=3.84$

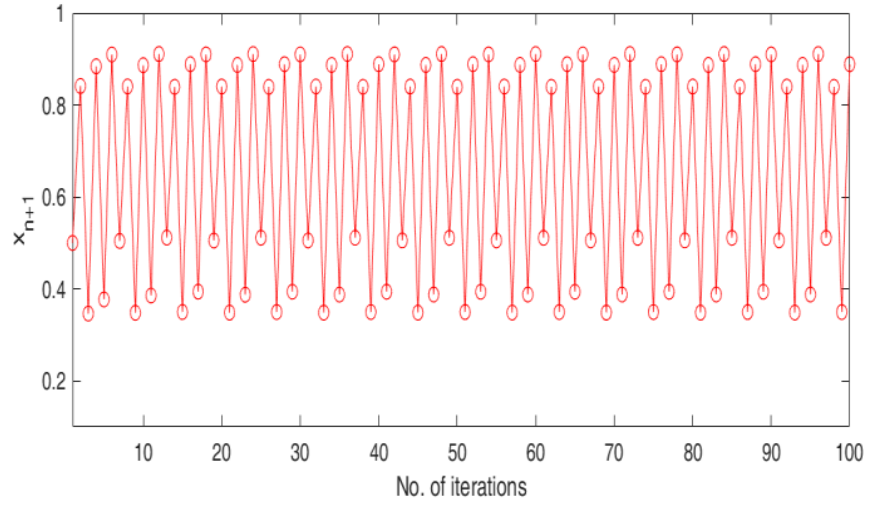

Fig. 7. Orbit of order-6 at $\lambda=\mu=v=0.9, r_{1}=3.823$ and $r_{2}=3.89$

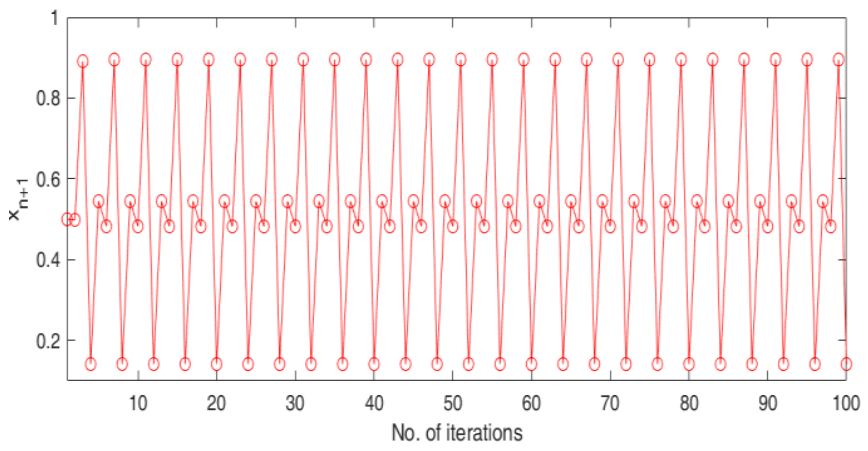

Fig. 8. $4^{\text {th }}$ order cycle at $\lambda=\mu=v=0.8, r_{1}=4.57$ and $r_{2}=4.02$

\section{CONCLUSION}

In the present work, the chaotic situation of logistic map has been controlled with the help of switching strategy (Parrondo's paradox) by adopting bifurcation and time series plots. The logistic map in SP orbit exhibits chaotic behavior for $r>3.85$ as shown in Fig. 1. By applying switching strategy, the two chaotic orbits may result into a periodic orbit (see Figs. 5, 6, 7 and 8), i.e., chaotic orbit $1+$ chaotic orbit 2 = periodic orbit. Thus, one can control the chaos of dynamical systems by applying switching strategy. Moreover, our approach of chaos and bifurcation analysis can be used in signal masking and in stability analysis of power system networks.

\section{REFERENCES}

1. P.F. Verhulst, "Recherches mathmatiques sur la loi d'accroissemen de la population," Nouveaux Mmoires de l'Acadmie Royale des Sciences et Belles-Lettres de Bruxelles, vol. 18, 1-42, 1945.

2. H. Peitgen, H. Jurgens, D. Saupe, "Chaos and Fractals," Springer Verlag, New York, 2004.

3. R.L. Devaney, "A First Course in Chaotic Dynamical Systems: Theory and Experiment," Addison-Wesley, Boston; 1992.

4. J.M.R. Parrondo, "How to cheat a bad mathematician," EEC HC \& M network on complexity and chaos, Italy: ISI, Torino; 1996. Unpublished

5. G.P. Harmer, D. Abbott, "Game theory: losing strategies can win by Parrondo's paradox," Nature, 402-864, 1999.

6. G.P. Harmer, D. Abbott, "A review of Parrondo's paradox," Fluc Noise Lett., vol. 2, R71-R107, 2002.

7. D. Abbott, "Asymmetry and disorder: a decade of Parrondo's paradox,” Fluc Noise Lett., vol. 9, 129-156, 2010.

8. J. Almeida, D. Peralta-Salas, M. Romera, "Can two chaotic systems give rise to order?” Physica A, vol. 200, 124-132, 2005.

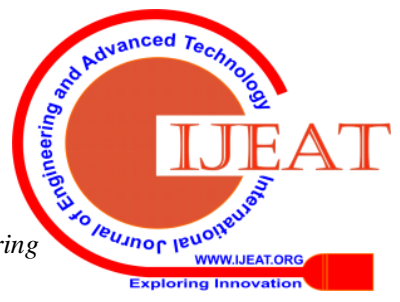


9. W. Fulai, "Improvement and empirical research on chaos control by theory of "chaos + chaos = order", Chaos, vol. 22, 43-145, 2012.

10. S. Kumari, R. Chugh, "A New Experiment with the Convergence and Stability of Logistic Map via SP Orbit,” Int. J. of App. Eng. Res., vol. 14, 797-801, 2019.

11. S. Kumari, R. Chugh, A. Nandal "Bifurcation Analysis of Logistic Map Using Four Step Feedback Procedure,” Int. J. Eng. Ad. Tech., vol. 9, 704-707, 2019.

12. E. Peacock-López, "Seasonality as a Parrondian game," Phys. Lett. A, vol. 375, 3124-3129, 2011.

13. E. Silva, E. Peacock-López, "Seasonality and the logistic map," Chaos Solitons Fractals, vol. 95, 152-156, 2017.

14. M. Rani, A. Yadav, A., "Parrondo's paradox in the superior logistic map", Int. Journal Tech. Research, vol. 1, no. 2, 1-8, 2016.

15. A. Yadav, K. Jha, "Parrondo's Paradox in the Noor Logistic Map", Int. J. Ad. Res. Eng. Tech., vol. 7, 01-06, 2016.

16. W. Phuengrattana, S. Suantai, "On the rate of convergence of Mann Ishikawa, Noor and SP- iterations for continuous functions on an arbitrary interval,” J. Comput. Appl. Math., vol. 235, 3006-3014, 2011.

\section{AUTHORS PROFILE}

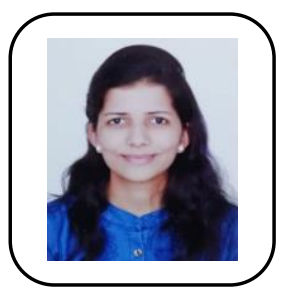

Mrs. Sudesh Kumari is working as an Assistant Prof. of Mathematics in Govt. College for Girls Sector 14, Gurugram.

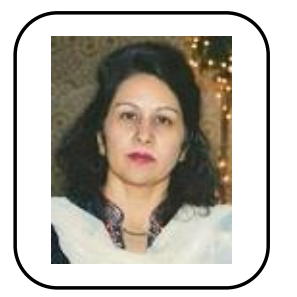

Professor. Renu Chugh is a Professor of Mathematics in Maharshi Dayanand University, Rohtak. She has 34 years research experience. About 190 research articles have been published by her in reputed national and international journals.

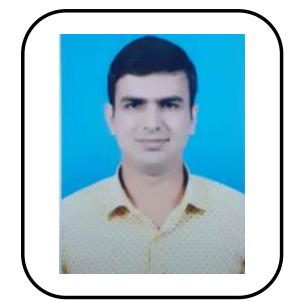

Mr. Ashish Nandal is working as an Assistant Professor of Mathematics in Pt. NRS Government College, Rohtak. 